\title{
Determinación de los Coeficientes de Transferencia de Masa Energía para el Proceso de Secado de Textiles, en Función de la Densidad del Material
}

\author{
Katiuska Franceschi, Sergio Pérez y Donato Magarelli \\ Facultad de Ingeniería, Escuela de Ingeniería Química, Departamento de Ingeniería Química, \\ Universidad de Carabobo, Estado Carabobo-Venezuela. (e-mail: kattyfg24@gmail.com, \\ sperez@uc.edu.ve,dmagarel@uc.edu.ve)
}

Recibido Nov. 04, 2009; Aceptado Ene. 04, 2010; Versión Final recibida Mar. 19, 2010

\begin{abstract}
Resumen
Se determinan los coeficientes de transferencia de materia y de energía presentes en el proceso de secado por contacto con una superficie caliente de textiles delgados. Se usaron cuatro textiles de diferentes densidades y datos experimentales del proceso de secado. Se determinaron los coeficientes para evaluar el comportamiento de cada tipo de material textil en función de la temperatura del secador cilíndrico. Los materiales textiles presentan densidades entre 144 y $230 \mathrm{~g} / \mathrm{m}^{2}$, y el comportamiento de los coeficientes de transferencia con respecto a la temperatura del cilindro, tienen similitud para los textiles con densidad menor a $200 \mathrm{~g} / \mathrm{m}^{2}$ presentando mayores valores del coeficiente de transferencia de energía y menores de transferencia de materia en comparación con los de mayor densidad.
\end{abstract}

Palabras clave: secado, coeficiente de transferencia de materia, coeficiente de transferencia de energía, textil

\section{Determination of the Coefficients of the Mass Transfer and of Energy for the Drying Process of Textile, in Function of the Density of the Material}

\begin{abstract}
The mass transfer coefficient and of energy coefficient by contact presents during the drying process of a thin textile were determined. Four textile material of different density and experimental data of the drying process were used. The coefficients were determined to evaluate the behavior of each type of textile material in function of the temperature of the cylindrical dryer. The textile materials present densities between 144 y $230 \mathrm{~g} / \mathrm{m}^{2}$, and the behavior of the transfer coefficients with respect to the temperature of the cylinder is similar to that found for textile with density smaller than $200 \mathrm{~g} / \mathrm{m}^{2}$ presented higher values of the heat transfer coefficients and lower values of the mass transfer coefficients, compared to the material of higher density.
\end{abstract}

Keywords: drying, mass transfer coefficient, heat transfer coefficient, textile 


\section{INTRODUCCIÓN}

En el proceso de secado de textiles existen técnicas innovadoras que utilizan electricidad en su principio de secado las cuales se caracterizan por un menor consumo de energía (Dhib, 2007). Un técnica más reciente es el empleo de inducción eléctrica para calentar un cilindro rotatorio sobre el cual, por contacto directo se elimina humedad de una fibra textil, teniéndose un menor consumo de energía en el proceso de secado, en comparación al proceso tradicional mediante empleo de vapor de agua (Fan y Wen, 2002, Pérez et al., 2001).

Para el desarrollo de modelos matemáticos de diferentes alternativas de secado de materiales porosos, se dispone en la literatura de diferentes ecuaciones matemáticas que pueden usarse para el modelado de operaciones de secado, las que fueron desarrolladas para equipos con diferentes principios de funcionamiento (contacto directo, conveccion, radiación), tomando solo el comportamiento del material húmedo y el mecanismo de secado utilizado en cada caso (difusión, capilar, evaporación, condensación, etc.) (Fan y Wen, 2002; Lee et al., 2002, Jang y Chiu, 2007; Lu y Shen, 2007). Ribeiro (1995), realizó una evaluación del secado de textiles proponiendo su modelaje y un estudio con data experimental. En contraste al presente trabajo el autor realizó el secado a nivel de laboratorio empleando aire caliente. Johann (2010), determinó el calor de desorción para materiales textiles planteando correlación entre la presión de vapor y el calor latente de vaporización para dos textiles (algodón y elastane). En el presente trabajo se realiza la selección y adecuación de ecuaciones para material algodón de diferentes porosidades y densidad. Feng et al., (2001), desarrollaron un modelo de transferencia de masa y calor del secado por combinación de calentamiento por microonda y conducido por capa (microwave heating and spouted- bed drying. MWSB) de manzanas cortadas en cubo, un material poroso higroscópico, para considerar la dependencia termodinámica de la humedad y la temperatura, el cual es validado con datos experimentales.

De Kuang (1994) desarrolló un modelo matemático para describir el proceso de secado de papel por infrarrojo en una planta piloto, el cual estudia varios fenómenos (transferencia de masa de vapor y agua, transferencia de calor por conducción, convección radiación). El modelo matemático se valida utilizando resultados experimentales y puede predecir la humedad y temperatura del papel durante el secado. Pérez et al., (2001), planteó el modelaje del secado por inducción electromagnética de una tela a base de algodón, mientras que Franceschi (2008), realizó el modelaje de un proceso de secado por inducción eléctrica, involucrando el coeficiente de transferencia de masa $(\mathrm{Kg})$ y el coeficiente de transferencia de energía por contacto $(\beta)$. El primero de los coeficientes está asociado a la tasa de evaporación de agua del material textil, mientras que el segundo corresponde a la facilidad de la transferencia de energía desde el cilindro caliente hacia el material textil húmedo. En el presente trabajo, se determinan los coeficientes de transferencia de masa y energía realizando un análisis de su comportamiento con respecto a la densidad que presentan cuatro materiales textiles a base de algodón que tienen diferencias en cuanto a su porosidad y estructura de entrelazado, como se muestra en la Fig. 1.

\section{DESCRIPCION DEL MONTAJE EXPERIMENTAL}

El proceso consiste en poner en contacto directo la tela con la superficie de un cilindro metálico rotatorio hueco cuya superficie es calentada por inducción eléctrica. Para el calentamiento se emplean tres inductores electromagnéticos cada uno de ellos está formado por dos bobinas ocupando un $18.5 \%$ de la superficie total del cilindro. Los inductores disponen de una potencia máxima de $11.7 \mathrm{~kW}$, que fue limitada a un $90 \%$ de su capacidad lo cual equivale a $10.5 \mathrm{KW}$. Para la rotación del cilindro se utiliza un motor de $1 \mathrm{hp}, 600 \mathrm{~V}$, con una velocidad máxima de rotación de 14.3 $\mathrm{rev} / \mathrm{min}$. La velocidad de rotación del cilindro puede ser ajustada. El material poroso es mantenido en contacto al cilindro por medio de dos rodillos R1 y R2, siendo R2 un rodillo de posición fija mientras que R1 puede tener distintas posiciones lo cual genera diferentes superficies de contacto entre el material poroso y el cilindro. Dependiendo de la posición angular del rodillo R1 es posible contar con una superficie máxima y mínima de contacto de $1621.5 \mathrm{~cm}^{2}$ y $471.5 \mathrm{~cm}^{2}$ respectivamente. En la Fig. 2 se puede apreciar la zona de secado además de las diferentes superficies correspondientes a cada sección del cilindro. 


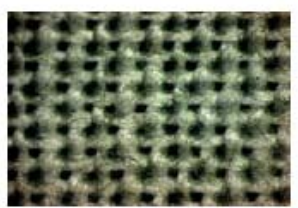

Textil tipo 1

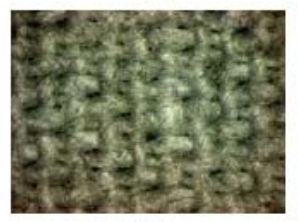

Textil tipo 3

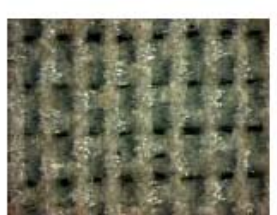

Textil tipo 2

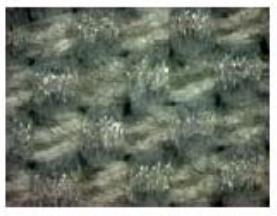

Textil tipo 4

Fig. 1: Fotos microscópicas de los diferentes textiles usados en los experimentos

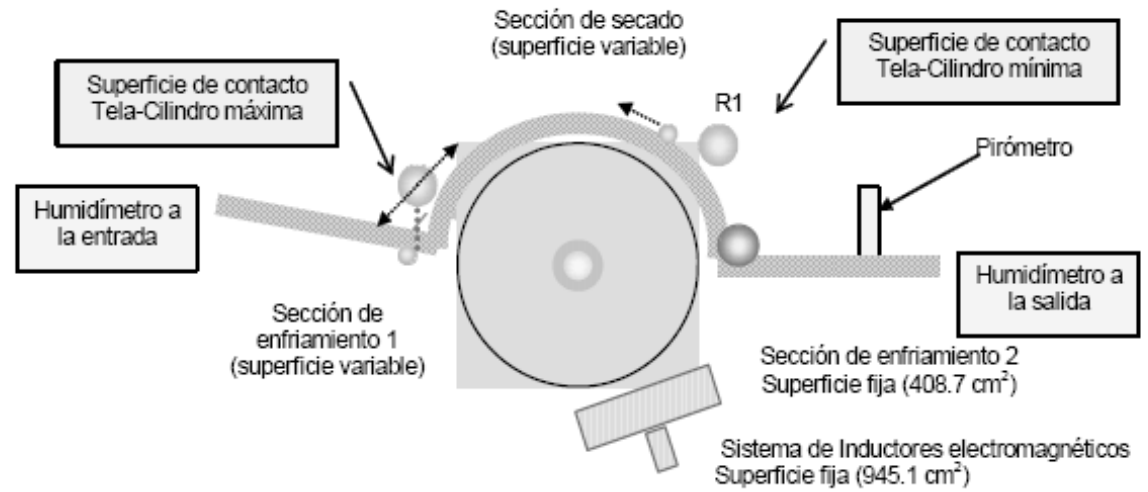

Fig. 2: Representación de distintas superficies del cilindro rotatorio.

\section{METODOLOGIA}

Se disponen de cuatro textiles de uso comercial, todos son de base de algodón que presentan diferencias en cuanto al entrelazado de las fibras de cada material. A cada textil se le determinó su masa medida en una balanza granataria digital marca Gibertini modelo TM4600 AR (max 4000g $\mathrm{d}=0.1$ y $\max 600 \mathrm{~g} \mathrm{~d}=0.01 \mathrm{~g}$ ). Luego al trozo de material se le calculó el área y de esta manera se cuantificó la densidad superficial $\left(\mathrm{g} / \mathrm{m}^{2}\right)$, correspondiente a cada una de las cuatro muestras disponibles para el desarrollo de la investigación.

El material textil presenta una superficie de contacto con el cilindro de $1621.5 \mathrm{~cm}^{2}$. Para el desarrollo del proceso experimental se fijó 1,90 rev/min como velocidad de rotación del cilindro y potencia de inducción de $7.02 \mathrm{~kW}$.

Basándose en balances de energía realizados en el cilindro, Franceschi et al.(2008), se disponen de los flujos de calor de convección $\left(\mathrm{Q}_{A}\right)$ y radiación $\left(\mathrm{Q}_{B}\right)$ del cilindro al medio y del cilindro a su parte interna $\left(Q_{c}\right.$ y $\left.Q_{D}\right)$, además del flujo de calor por conducción en la superficie metálica del cilindro $\left(Q_{E}\right)$. Se plantea el balance de energía en el cilindro con tela (Fig. 3), para determinar el flujo de energía por conducción $\left(\mathrm{Q}_{\mathrm{G}}\right)$ a partir de la ecuación 1 al disponer los datos de tiempo del proceso, dimensiones del cilindro y velocidad de rotación.

$\mathrm{m}_{\mathrm{c}} \mathrm{Cp}_{\mathrm{c}} \frac{\mathrm{dTc}}{\mathrm{d} \tau}=-\left[\left(\mathrm{Q}_{\mathrm{A}}+\mathrm{Q}_{\mathrm{B}}\right) \theta+\mathrm{Q}_{\mathrm{C}}+\mathrm{Q}_{\mathrm{D}}+\mathrm{Q}_{\mathrm{E}}+\mathrm{Q}_{\mathrm{G}}\right]$

Donde: $\mathrm{m}_{\mathrm{c}}$ : Masa total del cilindro $(\mathrm{kg}), \mathrm{Cp}_{\mathrm{c}}$ : Calor específico del material del cilindro, acero e igual a $8.214 \mathrm{~W}$ min $\mathrm{kg}^{-1}{ }^{\circ} \mathrm{C}^{-1}, \mathrm{~T}_{\mathrm{c}}$ : Temperatura del cilindro $\left({ }^{\circ} \mathrm{C}\right), \tau$ : Tiempo de enfriamiento (min.), $\theta$ : Fracción descubierta de la superficie superior del cilindro. 


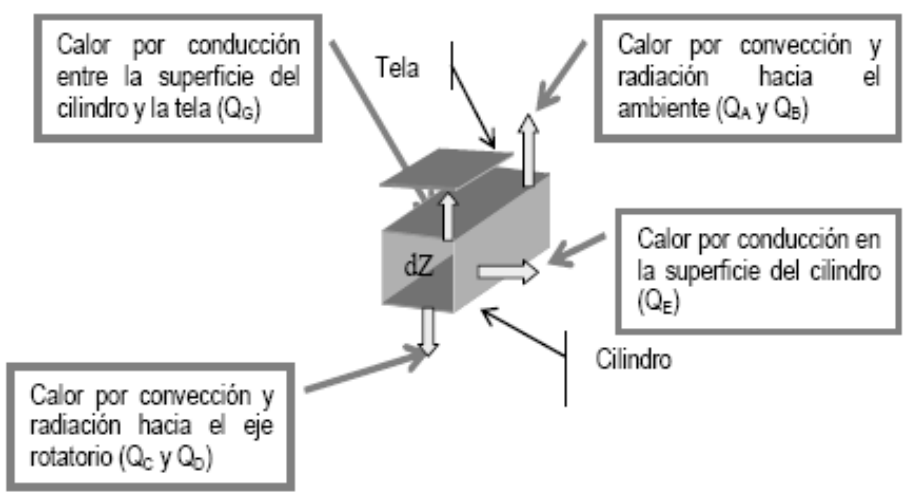

Fig. 3: Representación esquemática del proceso de secado para la aplicación del balance de energía

El proceso de secado se representa esquemáticamente para realizar el análisis de los flujos de calor presentes entre la tela y el cilindro, Fig. 4.

Se determina el calor convectivo desde la tela hacia el medio ambiente a partir de la ecuación 2.

$Q_{c t}=h L_{t} d Z\left(T_{t}-T_{\infty}\right)$

Donde: $Q_{c t}$ : Flujo de calor convectivo desde la tela hacia el medio externo $(\mathrm{W}), L_{t}$ : Longitud de la tela $(0.20 \mathrm{~m}), h$ : Coeficiente de transferencia convectiva $\left(\mathrm{W} \mathrm{m}^{-2}\right), \mathrm{dZ}$ : Diferencial de posición $\left(\mathrm{m}^{-1}\right)$.

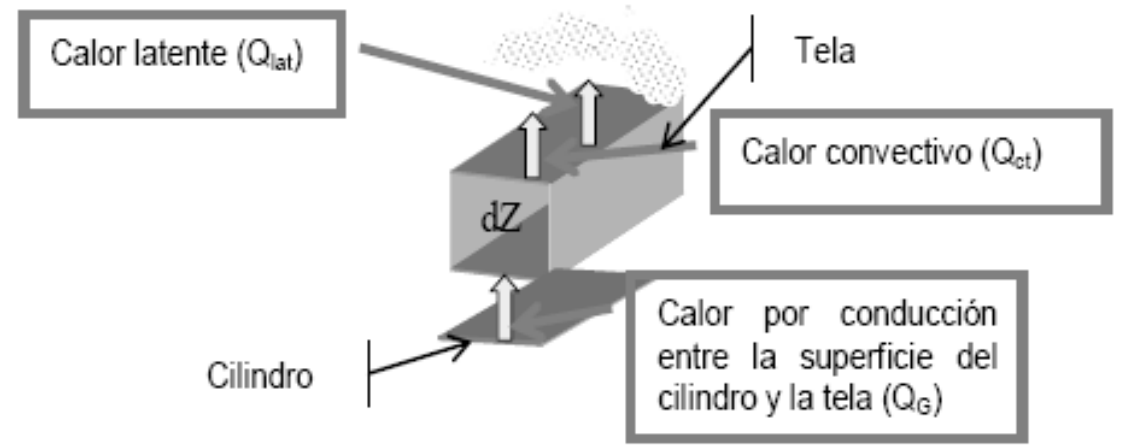

Fig 4: Representación esquemática del proceso de secado para la aplicación del balance de masa y energía fijando volumen de control la tela

Al plantear el balance de energía, considerando un volumen de control sobre la tela, se tiene la ecuación 3 que permite determinar el calor latente.

$m_{t h} C p_{t h} \frac{d T_{t}}{d \tau}=Q_{G}-Q_{l a t}-Q_{c t}$

Donde: $m_{t h}$ : Masa de tela húmeda $(\mathrm{g}), C p_{t h}$ : Calor específico de la tela húmeda (W min $\left.\mathrm{g}^{-1} \mathrm{o}^{-1}\right), T_{t}$ : Temperatura de la tela $\left({ }^{\circ} \mathrm{C}\right), Q_{\text {lat }}$ : Flujo de calor latente para la vaporización del agua $(\mathrm{W})$.

Determinado el calor latente se estima la tasa de agua evaporada a partir de la ecuación 4.

$M_{\text {evap }}=\frac{Q_{\text {lat }}}{h_{f g}}$

Donde: $h_{f g}$ : Calor de vaporización $\left(\mathrm{J} \mathrm{g}^{-1}\right)$ 
Al tener la tasa de agua evaporada se puede calcular el coeficiente de transferencia de masa (Kg) a partir de la ecuación 5.

$M_{\text {evap }}=\frac{\mathrm{KgL}_{\mathrm{t}} \mathrm{dZM}\left(\mathrm{P}_{\mathrm{v}}-\mathrm{P}_{\mathrm{a}}\right)}{\mathrm{RT}_{\mathrm{t}}}\left(\frac{\mathrm{H}_{\mathrm{t}}}{\mathrm{H}_{\mathrm{c}}}\right)^{\mathrm{n}}$

Donde: $\mathrm{Kg}$ : Coeficiente de transferencia de masa global $\left(\mathrm{m} \mathrm{s}^{-1}\right), M$ : Masa molar del agua (18 $\mathrm{g} \mathrm{mol}^{-}$ $\left.{ }^{1}\right), R$ : Constante de los gases ideales $\left(8.206 \times 10^{-5} \mathrm{~m}^{3}\right.$ atm $\left.\mathrm{mol}^{-1} \mathrm{~K}^{-1}\right), H_{c}$ : Humedad crítica de la tela $(\mathrm{g}$ de agua por $100 \mathrm{~g}$ de tela seca).

Al tener el flujo de calor por conducción $\left(\mathrm{Q}_{\mathrm{G}}\right)$, el coeficiente de transferencia de energía $(\beta)$ se determina mediante la ecuación 6.

$\mathrm{Q}_{\mathrm{G}}=\beta \mathrm{L}_{\mathrm{t}} \mathrm{dZ}\left(\mathrm{T}_{\mathrm{c}}-\mathrm{T}_{\mathrm{t}}\right)$

Donde $\beta$ : Coeficiente de contacto de conducción en la interfase entre la tela y el cilindro $\left(\mathrm{W} \mathrm{m}^{-2}{ }^{\circ} \mathrm{C}^{-1}\right)$

La modelación del secado se validó con data experimental del montaje del proceso, contándose con un promedio de 14 revoluciones por cada tela disponiéndose de valores experimentales de humedad de la tela a la salida del proceso y temperaturas que permiten verificar la buena predicción del modelo.

\section{RESULTADOS}

A los cuatro textiles de algodón se les determinó la densidad superficial, Tela 1: $144,4 \mathrm{~g} / \mathrm{m}^{2}$, Tela 2: $167,49 \mathrm{~g} / \mathrm{m}^{2}$, Tela $3: 228,49 \mathrm{~g} / \mathrm{m}^{2}$ y la Tela $4: 230,34 \mathrm{~g} / \mathrm{m}^{2}$, apreciándose que dos de ellas presentan densidades por debajo a $200 \mathrm{~g} / \mathrm{m}^{2}$ y las dos restantes valores superiores. El coeficiente de transferencia de masa $\left(K_{g}\right)$ sigue el comportamiento de la tasa de evaporación $\left(M_{\text {evap }}\right)$, mientras que el coeficiente de transferencia de energía $(\beta)$ tiene un comportamiento proporcional al flujo de calor de conducción $\left(Q_{G}\right)$ (Fig. 5 y Fig. 6). Por tal razón a partir de los coeficientes de transferencia de masa y de energía se puede predecir el comportamiento de la tasa de evaporación y el suministro de energía de conducción del cilindro a la tela.

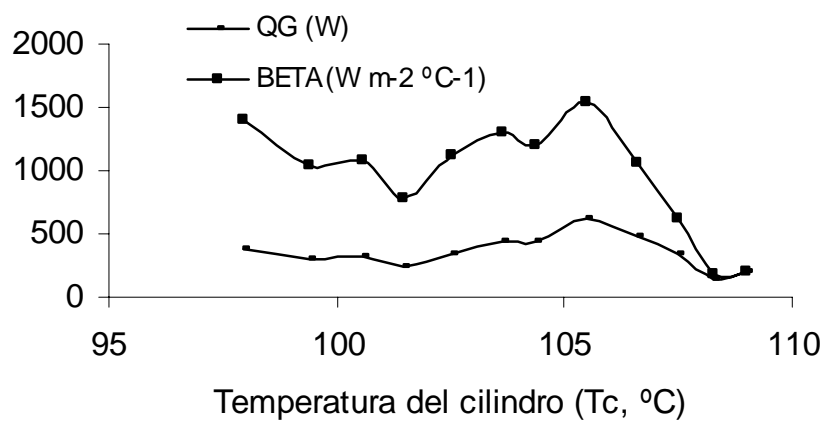

Fig.5: El flujo de calor de conducción $\left(\mathrm{Q}_{\mathrm{G}}\right)$ y Coeficiente de transferencia de energía ( $\beta$ ) para la Tela 2 a diferentes temperaturas del cilindro

Wilhelmsson et al., (1993), estudia el secado de diferentes tipos de papel. En su trabajo reporta valores del coeficiente de transferencia de energía entre 300 y $1400 \mathrm{Wm}^{-2}{ }^{\circ} \mathrm{C}^{-1}$. Mientras que en el estudio de Pérez et al., (2001) los valores del coeficiente de transferencia de energía se encuentran entre 300 y $1300 \mathrm{Wm}^{-2}{ }^{\circ} \mathrm{C}^{-1}$. En la Fig. 5, se aprecia que el coeficiente de transferencia de energía $(\beta)$ alcanza valores semejantes a los reportados por otros autores en procesos similares. Cui et al., (1999) estudia el secado de papel empleando flujo de aire transversal. Reporta valores del coeficiente de transferencia de masa entre 1.2 y $4.8\left(\mathrm{~m} \mathrm{~s}^{-1}\right)$. En este caso se aprecia en la Fig. 6 que los valores de $\mathrm{K}_{\mathrm{g}}$ son menores a los reportados por otros autores, ya que Cui et al., (1999) aplica una 
técnica de secado diferente a la empleada en la investigación actual que permite una mayor tasa de evaporación del agua.

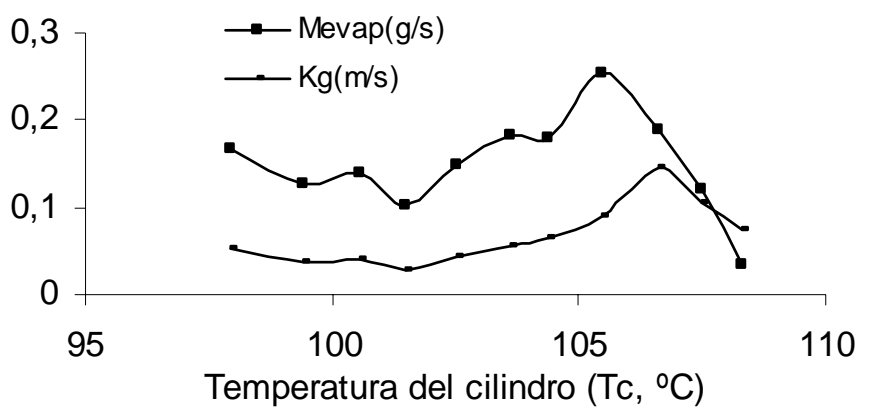

Fig.6: Tasa de evaporación $\left(\mathrm{M}_{\mathrm{evap}}\right)$ y coeficiente de transferencia de masa

$\left(\mathrm{K}_{\mathrm{g}}\right)$ para la Tela 2 a diferentes temperaturas del cilindro

En el secado de los textiles, en la medida en que avanza el proceso disminuye la temperatura del cilindro mientras que la temperatura de la tela aumenta (Fig. 7) ocasionando que la diferencia de ambas temperaturas disminuya, por tal razón el coeficiente de transferencia de energía ( $\beta$ ) aumenta, Fig. 8.

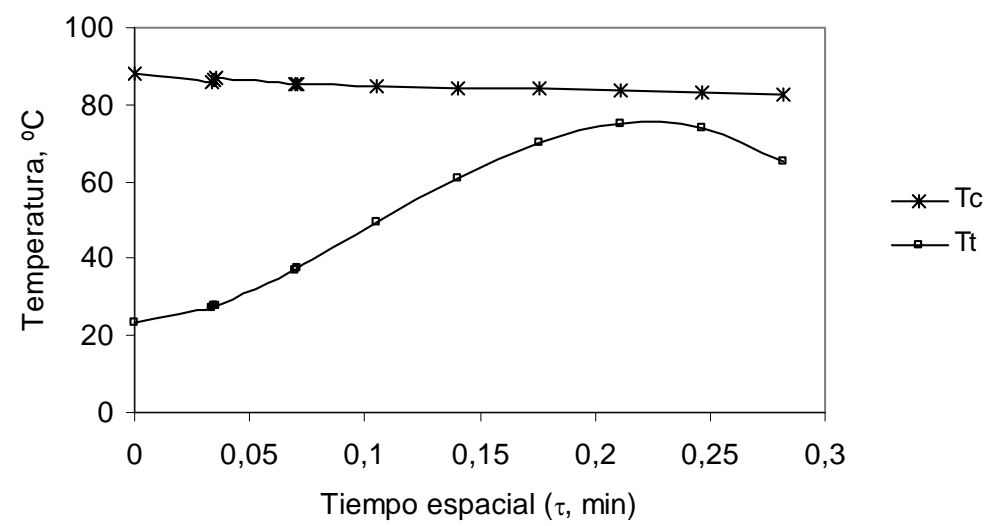

Fig.7: Perfil de temperatura del cilindro y de la tela 4 en el proceso de secado.

Los procesos de secado se dividen en tres fases. En la primera, la tasa de agua evaporada presenta un leve incremento. En la segunda se mantiene constante para finalizar con una disminución de la misma, Pérez et al., (2000). En tal sentido, se puede explicar el comportamiento que se observa en la Fig. 8, al disminuir la tasa de agua evaporada se produce un decrecimiento en el comportamiento del coeficiente de transferencia de masa $\left(\mathrm{K}_{\mathrm{g}}\right)$.

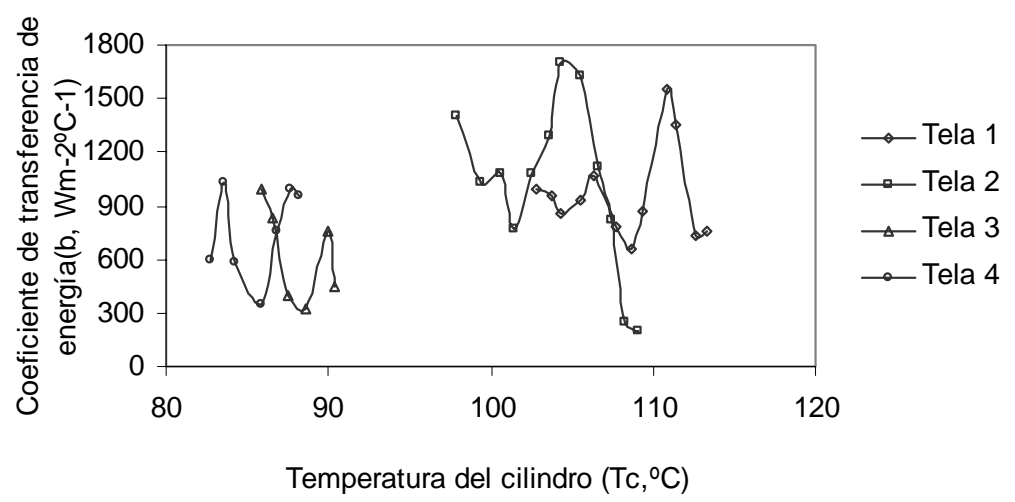

Fig. 8: Coeficiente de transferencia de energía de los cuatro textiles en función de la temperatura del cilindro en el proceso de secado 


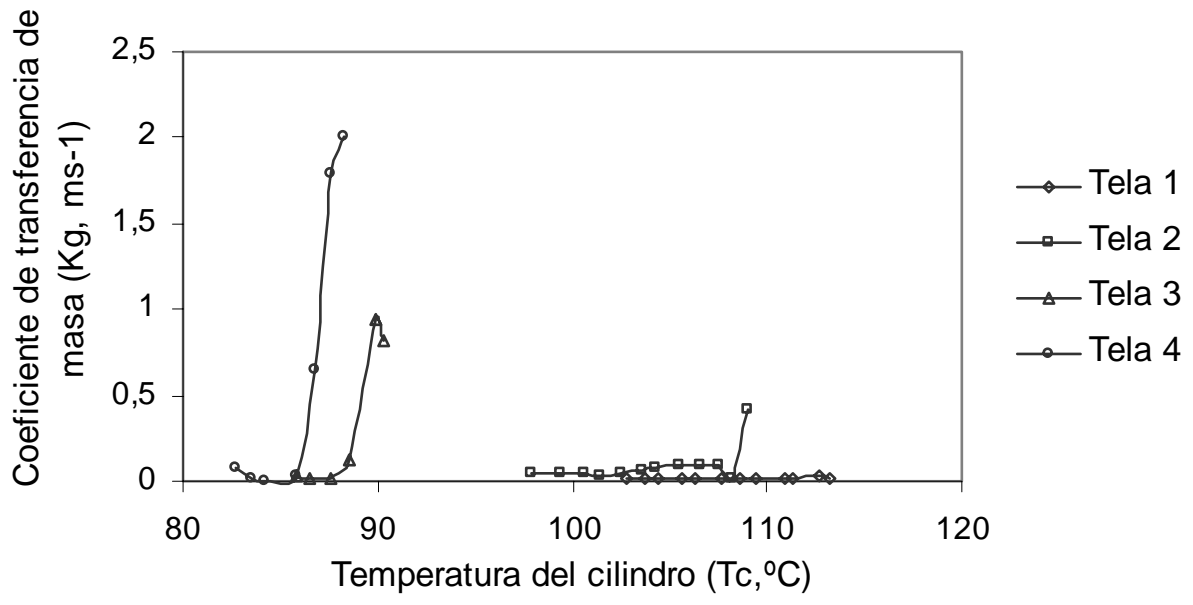

Fig. 9: Coeficiente de transferencia de masa de los cuatro textiles en función de la temperatura del cilindro en el proceso de secado

Analizando las Figs. 8 y 9, se puede apreciar que las Telas 1 y 2, las cuales presentan densidades inferiores a los $200 \mathrm{~g} / \mathrm{m}^{2}$ tienen un comportamiento similar entre ellas en cuanto a los coeficientes estudiados. De igual manera sucede con las Telas 3 y 4 que tienen densidades superiores. Las Telas 1 y 2 presentan un tejido menos entrelazado en comparación a las Telas 3 y 4 , (ver Fig. 1), por ello presentan la diferencia entre sus densidades y a la vez permite explicar la similitud en el comportamiento de los coeficientes involucrados en el secado del material textil. Mientras menos entrelazado es el tejido se tiene un secado a temperaturas del cilindro mayores que en el caso contrario, contribuyendo a presentar mayores diferencias entre la temperatura del cilindro y de la tela, de esta manera se requiere un mayor calor por conducción $\left(\mathrm{Q}_{\mathrm{G}}\right)$ lo que ocasiona mayores valores para el coeficiente de transferencia de energía $(\beta)$.

En lo que corresponde a la tasa de evaporación de agua será menor para tejidos menos entrelazados ya que presentan mayor dificultad para el proceso de secado, lo que implica menores valores de los coeficientes de transferencia de masa $\left(\mathrm{K}_{\mathrm{g}}\right)$ para las Telas 1 y 2 . Por las características del textil y la porosidad, las Telas 3 y 4 presentan una mayor facilidad para alcanzar el incremento de la tasa de evaporación en comparación a las Telas 1 y 2, la cual se obtiene luego de un tiempo espacial requerido para alcanzar la estabilidad del proceso. En la parte final del proceso de secado en las telas se produce una disminución de la tasa evaporada debido a que presentan zonas secas en gran parte de la tela.

\section{CONCLUSIONES}

Las Telas 1 y 2 (densidades inferiores a los $200 \mathrm{~g} / \mathrm{m}^{2}$ ) tienen un comportamiento similar entre ellas presentando mayores valores del coeficiente de transferencia de energía en comparación a las Telas 3 y 4 , por requerir menor calor por conducción. En cuanto al coeficiente de transferencia de masa será menor para las Telas 1 y 2 que tienen mayor dificultad para secarse presentando una menor tasa de evaporación con respecto a las Telas 3 y 4 . . El comportamiento de los coeficientes de masa y energía se siguen por la variación de la tasa evaporada y el calor de conducción generado a la tela a partir de la superficie del cilindro.

\section{REFERENCIAS}

Cui, Y., S. Ramaswamy y C. Tourigny; Through-air drying of tissue and towel grades. Tappi J. 82, 203-208 (1999).

De Kuang, H., Le séchage du papier par infrarouge. Thèse doctorat, Université Laval. Canadá, (1994). 
Dhib, R.; Infrared Drying; From Process Modeling to Advanced Process Control, Drying Technology, 25(1), $97-105$ (2007)

Fan J. y X. Wen; Modeling Heat and Moisture Transfer Through Fibrous Insulation with Phase Change and Mobile Condensate, International Journal of Heat and Mass Transfer: 45, 4045-4055 (2002).

Feng H., Tang J., Cavalieri R., Pluma O., Heat and mass transport in microwave drying of porous materials in a spouted bed. AIChE Journal, 47,N07,1499-1512 (2001).

Franceschi, K., S. Pérez, Z. Niño y D. Magarelli; Modelado y Simulación del Secado de Textiles Delgados Mediante un Cilindro Calentado por Inducción Eléctrica. Inf. tecnol., ISSN 0718-0764, 19(4), 35-46. http://www.citchile.cl (2008).

Jang, J. Y. y Y. W. Chiu; Numerical and Experimental Analysis for a Metallic Hollow Cylinder Subject to Step-wise Electro-magnetic Heating, Applied Thermal Engineering: 27, 1883-1894 (2007).

Johann G., Determining heat of desorption for textile materials, Acta Scientiarum-Technology: 32, 63 (2010).

Lee, H.S., W. W. Carr, H. W. Beckham y J. Leiden; A Model of Through-air Drying of Tufted Textile Materials, International Journal of Heat and Mass Transfer: 45, 357-366 (2002).

Lu, T. y S.Q. Shen; Numerical and Experimental Investigation of Paper Drying: Heat and Mass Transfer with Phase Change in Porous Media, Applied Thermal Engineering: 27, 1248-1258 (2007).

Pérez, S., Therien, N., Broadbent, A.D., Modelling the continuous drying of a thin sheet of fibres on a cylinder heated by electric induction. The Canadian Journal of Chemical Engineering, 79, No6, 977989(2001).

Ribeiro J. y Ventura J.; Evaluation of textile bobbins drying processes-experimental and modelling studies, Drying Technology: 13, 239-265. (1995).

Wilhelmsson, B., L. Nilsson, S. Stenstrom y R. Wimmerstedt; Simulation models of multi-cylinder paper drying. Drying Techn. 11, 1177-1203 (1993). 